suivre l'évolution de la population; il repose sur deux méthodes. $1^{\circ}$ Une nomenclature générale des situations d'identité : chaque classe de gènes identiques porte un nom; pour un ensemble de gènes on définit les " états d'identité " où chaque gène est décrit par le nom de la classe à laquelle il appartient; une situation d'identité groupe des états d'identité qui ne diffèrent que par les classes de gènes identiques qui interviennent. Dans les calculs une situation d'identité est désignée par un état d'identité qui la représente. $2^{\circ} \mathrm{L}$ 'analyse séquentielle du pedigree : à partir des données, pour chaque individu, de son nom, du nom de son père et du nom de sa mère, le programme construit une suite de pseudo-générations. Chacune est telle que si l'on connalt les coefficients d'identité relatifs à elle, on peut calculer les coefficients d'identité relatifs à tout ensemble de zygotes qui descendent de zygotes de cette pseudo-génération. Une pseudo-génération $\left(G^{\prime}\right)$ se déduit de la précédente $(G)$ par introduction d'un fils ou d'une fille de $(G)$ et par exclusion de 0,1 ou 2 parents sans autre enfant. A chaque pas, on construit les situations d'identité qui résultent pour $\left(\mathrm{G}^{\prime}\right)$ de celles de $(\mathrm{G})$; on calcule leurs probabilités. De celles-ci on peut déduire les coefficients d'identité, de parenté, de consanguinité pour tout ensemble de gènes désigné à l'avance.

Le programme, écrit en langage Fortran IV, est d'ores et déjà fonctionnel : un pedigree de taille importante peut être traité par tranches de mille individus. L'exploitation de cette méthode de calcul sur des populations réelles permettra d'entreprendre la mesure de " distances génétiques " entre groupes d'individus apparentés, le calcul des covariances génotypiques dans les cas généraux, en outre, on pourra utiliser l'index de sélection établi par Rouvier (1969, Contribution à l'étude des index de sélection sur plusieurs caractère. Thèse $3^{\mathbf{e}}$ cycle, Fac. Sci. Paris).

\title{
VÉRIFICATION DE LA LOGIQUE INTERNE DE LA THÉORIE DE LA CONTRAINTE PAR DES EXPÉRIENCES ANALOGIQUES
}

J. Croizé-Pourcelèt. - Station centrale de Génétique animale, C.N.R.Z., 78-Jouy-en-Josas.

Après la Relation d'Identité (Grllors M., 1964. La Relation d'Identité en Génétique, Thèse Fac. Sci. Paris), GrLlors introduisit en 1965 la notion de Contrainte (GrLlors M., 1965. La Relation de Dépendance en Génétique. Journ. Féd. europ. Zootech. La Haye). Ce concept, plus subtil que les précédents, permet de traiter de nombreuses questions de génétique des populations, particulièrement celles ou intervient l'action des gènes. Il importe cependant, avant d'utiliser largement cette théorie nouvelle, d'en vérifier : $1^{\circ}$ la cohérence interne, c'est-à-dire la rigueur logique de sa conduite, et $2^{\circ}$ la cohérence externe, qui est la précision avec laquelle elle recouvre la phénomène étudié. Mais, dans l'état actuel de son développement, cette théorie n'est rigoureuse que si des hypothèses, dont la plus restrictive est l'absence de sélection, sont strictement observées. Comme il n'existe pas de population qui les vérifie constamment de façon certaine, il est impossible de tester sa cohérence interne au moyen d'expériences biologiques classiques. Si, en effet, les résultats de telles expériences différaient des prévisions théoriques, on ne pourrait savoir si ces différences sont dues à une erreur logique dans la théorie ou à l'inobservation des hypothèses restrictives par les populations réelles. Pour vérifier la cohérence interne de la théorie, il faut donc réaliser des expériences parfaites à l'aide de populations de zygotes théoriques qui observent rigoureusement les hypothèses fondamentales, et dont les événements aléatoires soient tous déterminés par un processus certainement aléatoire. Si les résultats de telles expériences analogiques ne coïncident pas avec les prévisions théoriques, la preuve d'une erreur logique dans la théorie sera faite; s'ils coïncident au contraire. on pourra admettre que la théorie est juste.

Des expériences analogiques simples avec des pseudo-populations pratiquant l'homogamie génotypique partielle ont montré que la définition même de celle-ci portait une contradiction et 
permis d'établir que tout mode d'accouplement dans les populations limitées est en réalité défini par deux lois : $1^{\circ}$ celle qui préside au tirage des géniteurs, $2^{\circ}$ celle qui règle leurs rapports sexuels. Seule l'une des deux peut être définie a priori, l'autre est conditionnée par la première (CrorzePourcelet J., 1968. Étude théorique et expérimentale de l'Évolution de Petites Populations par la Notion de Contrainte. Thèse Sc. appl. Orsay). D'autres expériences analogiques plus complexes ont montré qu'il fallait connaître non seulement la probabilité $a$ prior $i$ " $\mathrm{p}$ " de tirer un gène, mais aussi les probabilités conditionnées de tirer ce gène, sachant qu'il provient d'un zygote dans un état de Contrainte donné. Repris dans cette optique nouvelle, les calculs aboutissent à des courbes d'évolution qui coïncident remarquablement avec les résultats de toutes les expériences analogiques réalisées depuis. On peut, par conséquent, admettre que la théorie de la Contrainte est rigoureusement logique. Avant de l'utiliser largement, il ne reste donc qu'à tester sa cohérence externe.

\section{EXEMPLE D'UTIIISATION DE LA THÉORIE DE LA CONTRAINTE : INTRODUCTION D'UN CARACTÈRE MONOFACTORIEL, DANS DES POPULATIONS DE DROSOPHIIES}

J. Croizḱ-Pourcelet. - Station centrale de Génétique animale, C.N.R.Z., 78-Jouy-en-Josas.

Des preuves de la cohérence interne de la théorie de la Contrainte introduite par GiLlois en 1965 (Grllors M., 1965. La Relation de Dépendance en Génétique. Journ. Fèd. europ. Zootech. La Haye) et (Gillors M., 1965. La Relation de Dépendance entre gènes non identiques. Journ. Féd. europ. Zootech. La Haye), et partiellement modifiée en 1967 après les expériences analogiques, ont été données par Croizé-Pourcelet en 1968 (Croizé-Pourcelet J., 1968. Étude théorique et expérimentale de l'Évolution de petites Populations par la Notion de Contrainte Thèse Sc. appl. Orsay). Il reste à montrer: $1^{\circ}$ ) que cette théorie décrit effectivement le phénomène biologique étudié, c'est-à-dire que les populations véritables ont l'évolution que prévoient les équations dans la mesure où elles vérifient les hypothèses pour lesquelles cette théorie est rigoureuse, $\left.2^{\circ}\right)$ qu'elle est concrètement utilisable. Dans une expérience type réalisée sur drosophiles, on introduit le caractère brown ( $b w$.; chr. II) dans dix populations cinnabar ( $c n$.; chr II) indépendantes et limitées, en accouplant systématiquement à chaque génération 50 p. 100 des femelles reproductrices, choisies au hasard, à des mâles $b w$ purs exogènes. Les conditions expérimentales, choix des gènes, nombre de reproducteurs, pourcentage de femelles accouplées à des mâles $b w$, temps de copulation et de ponte, ont été définis de façon à approcher au plus près les hypothèses fondamentales de la théorie, et après une étude quantitative de la fécondité relative des différents types de couples, démontrant que les effets de la sélection différentielle sont négligeable en première approximation. Les courbes d'évolution calculées en utilisant la théorie de la Contrainte et celles représentant la moyenne des structures de ces dix populations indépendantes sont voisines sans se superposer complètement. Par exemple, à la $3^{\mathrm{e}}$ génération, les valeurs théoriques de P, R, $2 Q$ sont respectivement 16 p. 100,32 p. 100, 52 p. 100; les valeurs obtenues chez les mâles sont $19,5 \%, 24$ p. $100,56,5 \%$; à la $4^{\mathrm{e}}$ génération, les valeurs théoriques sont 9 p. 100,46 p. 100, 45 p. 100 et les valeurs expérimentales : $11,5 \%, 42$ p. 100, 46,5\%. Comme les expériences analogiques ont prouvé la cohérence interne de la théorie, on doit conclure que les écarts observés maintenant sont dus à une sélection résiduelle. En raison cependant du nombre faible d'expériences réalisées et de l'absence de connaissances théoriques sur les moments du second ordre que nous savons seulement minorer, on ne peut parler que de vérification au premier ordre de la qualité de la description des phénomènes biologiquement étudiés par la notion de Contrainte. La réalisation de cette expérience enfin donne un exemple d'utilisation schématique mais concret d'utilisation des coefficients de Contrainte. 